\section{SEMBLANCE RESIDUAL MOVEOUT ANALYSIS TO FIND ERORRS AND UPDATING THE INTERVAL VELOCITY MODEL MODE IN THE HORIZON BASED TOMOGRAPHY METHOD}

\author{
Sudra Irawana*, Siti Noor Chayatia, Sismantob
}

aGeomatics Engineering Study Program, Departement of Informatics Engineering, Politeknik Negeri Batam, 29461, Batam, Indonesia

bGeophysical Study Program, Faculty of Mathematics and Natural Science, Universitas Gadjah Mada, 55281, Yogyakarta, Indonesia
Article history

Received

29 January 2020

Received in revised form

12 October 2020

Accepted

14 October 2020

Published online

27 October 2020

*Corresponding author sudra@polibatam.ac.id

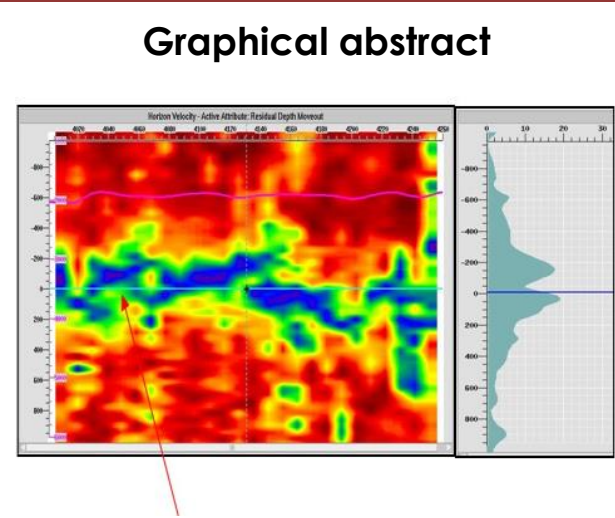

Axis of zero residual moveout

\begin{abstract}
The tomography method requires an excellent initial velocity model. On the horizon based tomography, it will correct the travel time error of seismic waves along the horizon which is analysed using input results from the analysis of residual depth moveout. In this study, a semblance residual moveout analysis will be conducted after the interval velocity model has applied to the SBI field seismic data (CDP Gathers and RMS velocity). Based on the imaging results generated by the PSDM running process, an aperture value of 550 for inline and 800 for crossline is selected. PSDM generated from the initial interval velocity model has an acoustic impedance value between $1000 \mathrm{~kg} / \mathrm{m}^{2} \mathrm{~s}$ to 14339.2 $\mathrm{kg} / \mathrm{m}^{2} \mathrm{~s}$. The PSDM process, residual moveout analysis, and horizon-based tomography are carried out iteratively until the error in the interval velocity model approaches zero. In this study, five iterations were performed. The resulting residual moveout is increasingly oscillating around zero after the 5th iteration, which indicates that the error in the interval velocity model is getting smaller. There are two types of residual moveout, namely residuals moveout positively and residuals moveout negatively. Residual moveout positive indicates that the velocity used is too high, while the residual moveout negative indicates that the velocity used is too low. The identification of interval velocity model errors with analysis of residual moveout semblance is calculated from depth gathers. The semblance residual moveout analysis is used for the Pre Stack Depth Migration (PSDM) depth image analysis stage along with the marker (well data). The incompatibility between depth image maps and reflectors that are related to the boundary layer in the depth image can be seen when both are displayed in a section (overlay), that is, the layer boundary at the interval velocity model will deviate from the reflector (layer boundary) it represents at the resulting depth image. Other errors can be identified from well markers that have not been tied to the horizon. Improvement of the interval velocity model is made iteratively until the velocity interval, and depth image models are produced accordingly.
\end{abstract}

Keywords: Semblance residual moveout, interval velocity, tomography, iteration, PSDM

(C) 2020 Penerbit UTM Press. All rights reserved 


\subsection{INTRODUCTION}

The subsurface imaging resulting from time migration does not consider the distortion of wave rays such as wave rays which are not straight lines. The result is influenced by the use of Root Mean Square (RMS) velocity on time migration which only serves to determine the overall wave propagation. The RMS velocity is not able to show the velocity of each subsurface in detail [1]. The way to obtain the interval velocity is by converting the velocity of RMS to interval velocity [2]. There are four methods to estimate the layer velocity which are based on ray tracing and travel time interactions, namely Dix transformation from RMS velocity, stacking velocity inverse, coherency inversion, and images gather analysis of the results of PSDM [3]. The Dix transformation method requires RMS velocity, the formulation used:

$$
v_{n}=\sqrt{\frac{V_{n}^{2} \tau_{n}-V_{n-1}^{2} \tau_{n-1}}{\tau_{n}-\tau_{n-1}}}
$$

Where $w_{n}$ is interval velocity of each layer limited by layer $(n-1)$ and layer $n, \tau_{n}$ and $\tau_{n-1}$ are two-way travel times for zero offset data, $V_{n}$ and $V_{n-1}$ are the RMS velocitys associated with each layer. The Dix equation is based on the equation that the boundary layer is flat and the offset used to calculate the velocity of RMS $V_{n}$ dan $V_{n-1}$ is in the low range [3].

In the ray tracing method, the rays are tracked to search for a subsurface location that is close to the actual geological conditions [4]. Thus, in in this process required a model of seismic interpretation that has been carried out with time migration. This method takes into account the bending of the wave ray as long as the wave of light propagates from the source to the subsurface towards the geophone. The stages of the ray tracing method include: stacking velocity inversion, coherency inversion, and image gather analysis of the results of PSDM [5]. Stacking velocity inversion requires time horizon taken from CMP-stacked data that has not been migrated and stacking velocity at the location analysed. Coherency inversion also requires time horizon taken from CMP-stacked that has not been migrated, does not require stacking velocity but analyses CMP gathers.

Velocity modelling requires several inputs related to the results of the interpretation. There are four input needed, namely lithology boundary, initial velocity and velocity range, local slope information, and markers identification in well data and seismic data [6]. Lithological boundaries are obtained from the interval velocity model of units that are layered with the boundaries of each layer which have differences or contrast of velocity value. The biggest velocity contrast in the geological layer is the boundary layer required at the interval velocity model. Knowledge of the initial velocity for each geological layer is at least a useful input for modelling the initial interval velocity
[7]. This information is obtained through downhole information, lithology, or through properties associated with velocity changes. This local angle slope is needed during the reflection tomography and PSDM process [8]. In addition to this, the input parameter requires the magnitude of the local slope of the study area. Identification of markers in the well data will be beneficial in the depth conversion process. This depth conversion process is essential to calibrate or validate the velocity model at the time of depth conversion [9]. Tying well data to the horizon is a standard task that needs to be done when producing a velocity model.

There are two types of tomography, namely horizon-based tomography and grid-based tomography [10]. Both are global approaches that can solve equations simultaneously to produce improved models of velocity and depth. On horizonbased tomography, what is analysed is the delay on each CRP along the path for each horizon on the model [11]. A grid-based tomography is an automatic approach with a small delay generated. Grid-based tomography is used in conditions when the tomography horizon cannot be used due to very complex structures, poor data quality, and reflectors on seismic cross-sections that are not continuous [12]. This tomographic method requires a good initial velocity model. This method also overcomes the problem based on statistics without conformity to the geological structure resulting in an erratic velocity model which eventually producing the wrong structure [13]. Horizon-based tomography will update the error of travel time for seismic waves along the analysed horizon [7]. This updating will result in the updating of depth errors and are expected to provide correct information about the subsurface velocity field. This method uses input results from the analysis of residual depth moveout [14]. Tomographic calculations are performed to update the slowness and time of wave propagation at the layer boundary.

Tomography using the residual moveout of the gathers pre-stack migration as an input for repairs with a depth error and offset will be changed to a time error along the CRP for the same offset. In determining the tomographic equation, a relationship between time errors, updated time models and slowness errors is needed. Conversion of depth error to time error when a signal at the border of a layer that is shifted vertically will be summed up as a depth error and the results of the change in time can be calculated [15]. The calculation of the tomography equation is solved by the weighted least square principle [16]. The relation between travel time and depth error can be seen using Nonparametric Statistics with the Brown-Mood Method in Multiple Linear Regression [17].

The velocity of a medium is influenced by various factors such as rock lithology, pressure, temperature, porosity, density, grain size, rock age, fluid content and frequency of wave propagation itself [18]. The velocity analysis used is the velocity spectrum analysis 
method, which is based on matching the velocity values for a particular seismic recording event. This value matching refers to the magnitude of the coherence value at the same point when the velocity value will be considered capable of representing a particular event if it has a high coherence value and vice versa. Coherence is a measure of mathematical similarity. Two data can be found for the similarity value by calculating the coherence. The greater the similarity between the two data, the greater the coherence value, that is, the value approaches one. The greater the difference between the two, the smaller the coherence value will be close to zero, and if the two data are increasingly opposite, the coherence value will be close to negative one [19]. The coherence criterion commonly used in velocity analysis is the semblance [20].

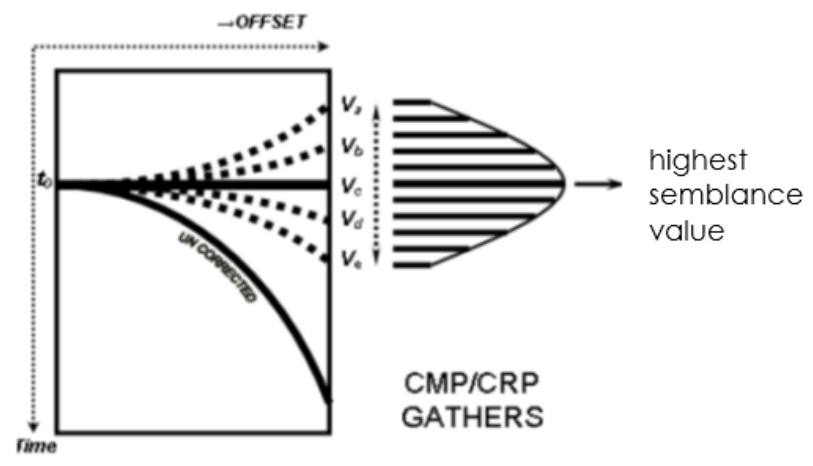

Figure 1 Coherence analysis with a semblance

Semblance analysis is used to find the velocity value based on the coherence value of the amplitude (Figure 1) with a high coherence value marked by a black spectrum assumed to be the velocity value that is most suitable for the related seismic event. In searching for seismic events, it must identify the seismic event that exists on the time record in the CMP gather data [14]. The next step is selecting the velocity value at the same location point through event identification on the CMP gather data [21].

In this research, the residual moveout Semblance analysis has been carried out after the interval velocity model has applied to the SBI field seismic data. If the interval velocity model has been estimated accurately, then the gathering depth that is generated from the Pre Stack Depth Migration (PSDM) with the interval velocity model shows flat events. The residual moveout analysis is performed to find errors at depth gathers due to the application of an inaccurate interval velocity model [22]. Errors in this interval velocity model can be seen in the residual moveout semblance which is still far from zero residual moveouts. This residual moveout is then used to update the interval velocity model. Analysis of residual moveout and the process of updating the velocity model is done iteratively until the velocity interval and depth image models produced are consistent or appropriate. Only a few iterations can achieve consistency for cases with small residual moves.

The research was conducted in the SBI field, which is one of Pertamina's exploration fields in Indonesia. Map of research location, with inline 2143 2323, and crossline 4001-4260, with four wells. There are 25 wells in the study area, but only use four wells, namely: well 1 , well 2 , well 3 , and well 4 . well 1 , well 3 , and well 4 are vertical wells, while well 2 is a sloping well at the same location as well 1.

\subsection{METHODOLOGY}

\section{A. Seismic Data}

The seismic data used in this study consisted of CDP gathers and RMS velocity. Details of each data are in Table 1.

Table 1 Seismic data

\begin{tabular}{|c|c|c|}
\hline No & Type & Information \\
\hline 1 & $\begin{array}{l}\text { CDP } \\
\text { Gathers }\end{array}$ & $\begin{array}{l}\text { Inline: } \\
\text { - first: } 2143, \text { last: } 2323 \text {, interval: } 24,99 \\
\text { m } \\
\text { Crossline: } \\
\text { - first: } 4001, \text { last: } 4260 \text {, interval: } 25,11 \\
\text { m }\end{array}$ \\
\hline 2 & $\begin{array}{l}\text { RMS } \\
\text { velocity }\end{array}$ & $\begin{array}{l}\text { - Inline: } 2143-2323, \text { crossline: } 4001 \text { - } \\
4260 \\
\text { - Maximum value: } 2605,58 \mathrm{~m} / \mathrm{s} \\
\text { - Minimum value: } 1670 \mathrm{~m} / \mathrm{s}\end{array}$ \\
\hline
\end{tabular}

\section{B. Well Data}

The wells in this study were 4, and each well was equipped with a well marker and log data. Well markers provide information about the depth of rock layers or rock formations in the study area. Usually, the data is used to determine layer boundaries, for example, top formations and bottom formations that are used for the processing and interpretation stages. Log data used include sonic log, density log, resistivity log, gamma ray (GR), and neutron log.

\section{Research Method}

The method used is the analysis of residual dept moveout to find errors and update the interval velocity model on the Horizon-Based Tomographic method. Tomography is a method used to improve the velocity model when PSDM is done with an inaccurate velocity model. Tomography uses measurements of residual moveout as inputs and attempts to find or create other models by removing errors that occur. The stages of research are presented in Figure 2. 


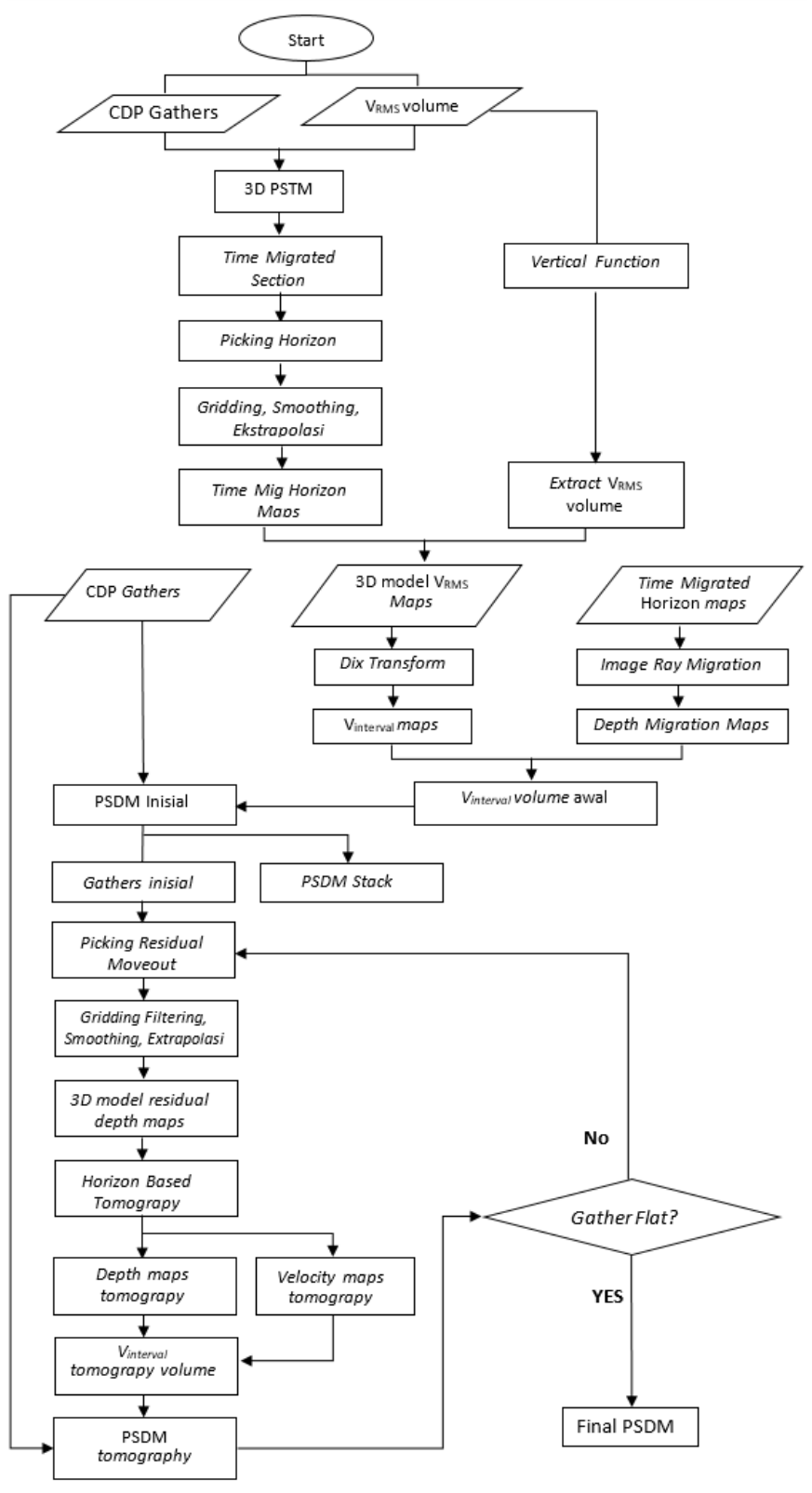

Figure 2 Research flow chart

\section{Running Pre-stack Depth Migration (PSDM)}

The input data needed for the PSDM process is the interval velocity 3D model, and the CDP gathers. The type of migration used in the study was 3D Kirchoof Pre-Stack Depth Migration. There were two stages in this process, namely: (1) calculation of travel time using the eikonal equation, (2) the migration process in the depth domain with the Kirchoff method. Several parameters must be included in this process, namely: inline and crossline targets, apartments, offset range, and types of input data (interval velocity models and CDP gathers) contained in seismic data managers.

The most influential parameter that must be determined in the process was the aperture. An aperture that is too small will make the data obtained under migrated because not all diffraction energies are summed well and also cause the migration process cannot provide maximum results because of the inaccessibility of the target reflector. Vice versa, if the aperture is too large, it will potentially cause noise and make the migration process last longer, and the file size will increase.

The basic principle of determining the aperture value is trial and error, but researchers have calculated the value with various variations, then analysed which aperture value provides the best imaging results. The basis for calculating the aperture value is as follows: (1) the inline and crossline values are first added with $25 \%$ of the initial inline and crossline values, so that the aperture value used is around 250 for inline, and 350 for crossline, (2) inline values and the first crossline is added with $50 \%$ of the initial inline and crossline values, so the aperture value used is around 300 for inline and 400 for crossline, (3) the inline and crossline values are first added by $100 \%$ of inline values and the initial crossline, so the aperture value used is around 400 for inline and 550 for crossline, (4) the inline and crossline values are first added with $200 \%$ of the initial inline and crossline values, so the aperture value is used around 550 for inline and 800 for crossline, (5) the inline and crossline values are initially added by $300 \%$ of the initial inline and crossline values, so the aperture value which is used around 750 for inline and 1050 for crossline.

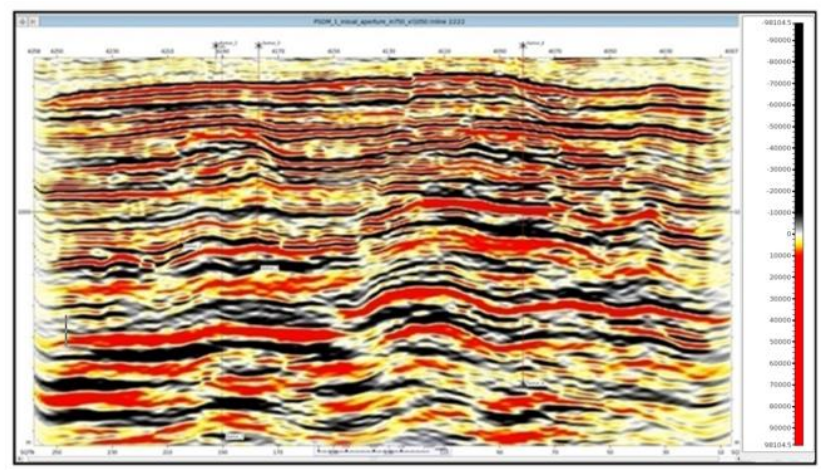

Figure 3 PSDM result with variations in the inline 550 and crossline 800 values

Based on the imaging results generated by the PSDM running process, an aperture value of 550 for inline and 800 was chosen for the crossline (Figure 3). The selection of this value is based on the continuity of the target reflector, the presence or absence of noise, the size of the file, and the length of the migration process. This aperture value will be used for the PSDM process then after being nominated. PSDM generated from the initial interval velocity model has an acoustic impedance value between $1000 \mathrm{~kg} / \mathrm{m}^{2} \mathrm{~s}$ to $14339,2 \mathrm{~kg} / \mathrm{m}^{2} \mathrm{~s}$. 


\subsection{RESULTS AND DISCUSSION}

\section{A. Residual Depth Moveout Analysis}

Analysis of residual moveout is carried out after an inaccurate interval velocity model is applied to seismic data. If the interval velocity model has been estimated accurately enough then the supposed depth gathering generated from PSDM with the interval velocity model shows events that are flat, but in reality, it is not, it can be seen there are still movesout, namely the difference in travel time between far-offset and near-offset as shown in Figure 4. This figure shows that there is still an error in the interval velocity model. This error can occur at the layer velocity or geometry reflector; therefore the next step is the residual moveout analysis.

The residual moveout analysis is performed to find errors at the collecting depth due to the application of an inaccurate interval velocity model. Errors in this interval velocity model can be seen in the residual moveout semblance which is still far from zero residual moveouts. This residual moveout is then used to update the interval velocity model. This analysis process begins by calculating the residual moveout semblance for all horizons in the velocity navigator window by assuming that the residual moveout is a parabolic function. Figure 5. shows the results of residual semblance that has been calculated on the first tomography (1st iteration) for all horizons.

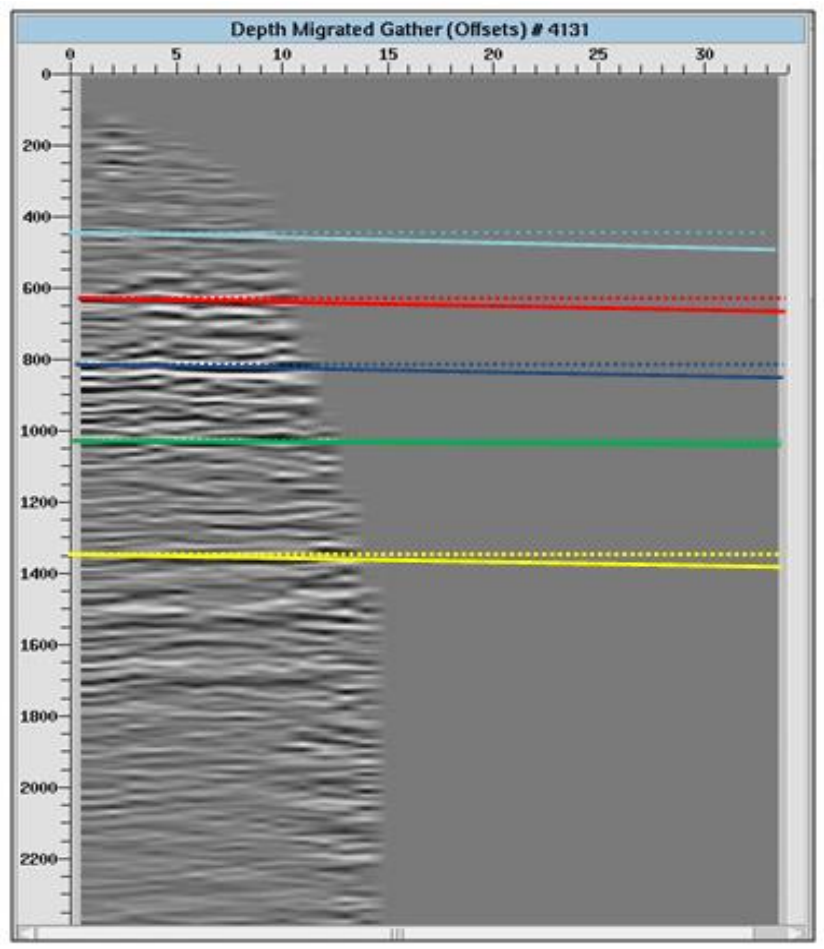

Figure 4 Gathers as the results of PSDM with the initial interval velocity model, it seems there are still events that are not flat

Based on Figure 5, it can be seen that the residual moveout semblance has two parameters related to the residual moveout positive and negative. Flat events produce a semblance peak that coincides with the horizontal axis (the green line in the semblance image) which shows zero residual moveouts. An event with a residual moveout will result in a semblance peak located above or below the horizontal axis depending on the size of the error. The deeper the layer, the maximum distribution of the semblance will be more random (irregular), this is due to the decrease in the $\mathrm{S} / \mathrm{N}$ ratio as the depth value increases so that the process of calculating the semblance value will be increasingly difficult.

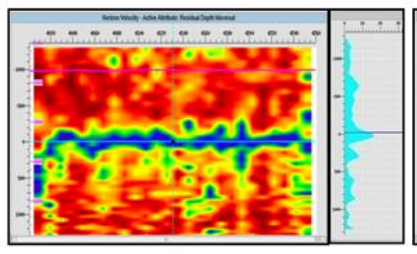

(a)

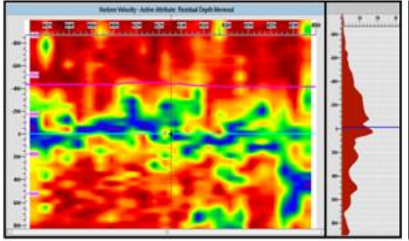

(c)

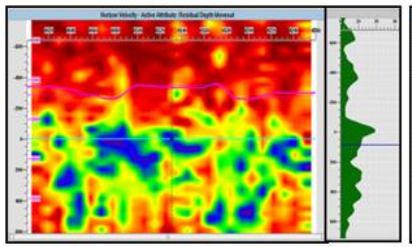

(e)

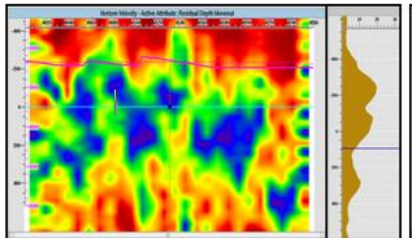

Figure 5 Semblance residual moveout after applying the initial interval velocity model, lst iteration tomography, (a) 1 horizon, (b) horizon 2, (c) horizon 3, (d) horizon 4, (e) horizon 5. (f) 6 horizon, (g) 7 horizon, (h) horizon 8

The next process after calculating the semblance for all horizons is picking semblance at the point with semblance which has a maximum value (semblance peak) and produces a flat gathering, and this can be seen in the QC depth gate panel (residual moveout analysis window). Picking semblance is done to find the sizeable residual moveout at each location of depth gathering along the horizon. In this study picking is done in 5 inline, starting from inline 2143 to inline 2323, this is done so that the detailed 
picking results are obtained, the meeting will be better, but requires a long time. Analysis of residual moveout and the process of repairing this velocity model is done iteratively until the velocity interval and depth image models produced are consistent or appropriate. Only a few iterations can achieve consistency for cases with small residual moves.

\section{B. Mapping Depth 3D Model Residual Moveout}

The next process after the residual moveout analysis, the picking residual moveout on the grid and a map of the residual moveout depth for all layers are analysed, then continued with map editing such as filtering, smoothing, and extrapolation for maps where there is still noise. Furthermore, maps of residual moveout depth are made in the form of 3D models by using a 3D model builder to become a map of the depth of the 3D residual moveout model. This map is used as input for improving the interval velocity model or as a substitute for depth maps for the new interval velocity modelling process. Figure 6. is an example of a map of the depth of the 3D residual moveout model generated after the first and fifth tomography for horizon 2.
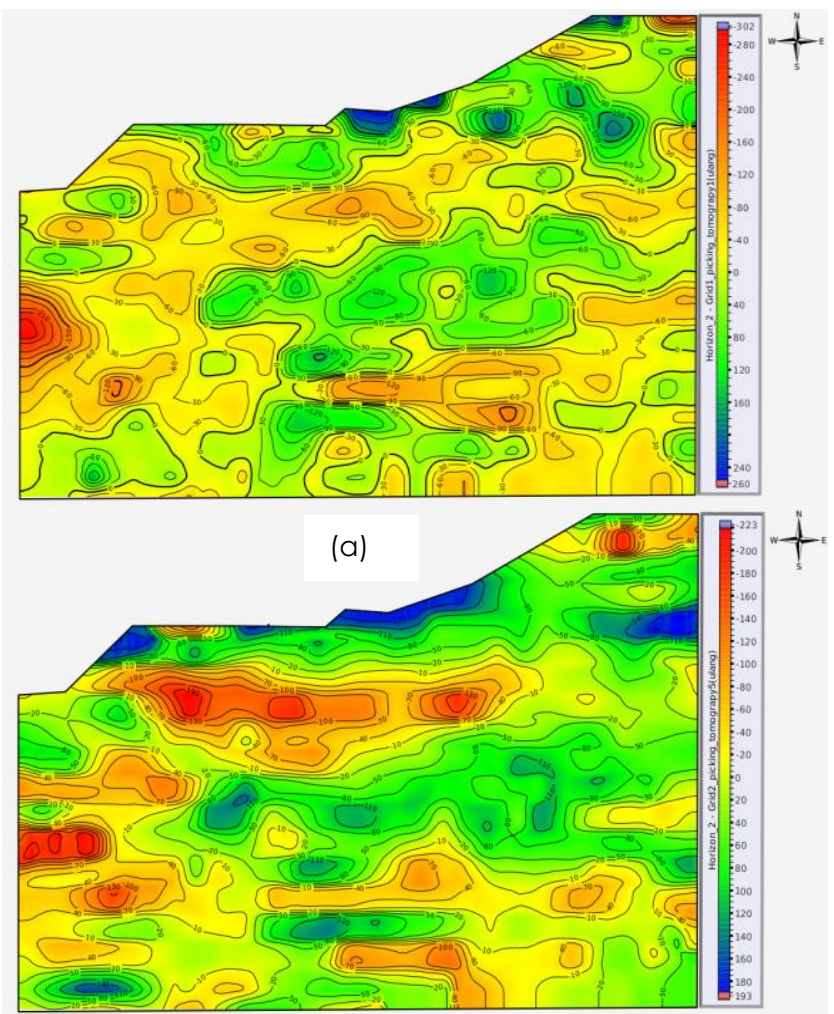

(b)

Figure 6 Map of 3D residual moveout depth model, residual moveout analysis results at horizon 2, (a) first iteration, (b) fifth iteration

\section{Horizon Based Tomography}

Map of 3D residual moveout depth models generated from the residual moveout analysis process is used as input for the tomography process using the Horizon-Based Tomographic method. This method calculates the travel time error used to update the interval velocity model and a depth map. One of the parameters that must be determined is a ray-tracing step, which shows the location of the origin of rays (ray-tracing starts or is traced from this point). In this study, the value of raytracing step 2 is used to increase the constraints in interpolating residual moveout values so that the results are expected to be more detailed.

The output of this tomography process is the interval velocity map (interval velocity maps) and depth maps for each horizon (there are nine horizons). Both of these maps have been updated with a smaller error than before, according to the purpose of this process which is to get new model parameters, slowness and reflector depth, so that the new interval velocity model is obtained. Interval velocity maps and depth maps are then editing that includes statistical analysis, filtering, smoothing and extrapolation, for each horizon so that a good map is obtained (free of noise), then a 3D model is created with a 3D model builder. The next process is to create a new volume velocity interval model with input interval 3D model maps and 3D model maps of depth for each horizon that has undergone an updating process. The final process is PSDM with CDP input and updated interval velocity models.

The PSDM process, residual moveout analysis, and tomography (horizon-based tomography) are carried out iteratively until the error in the interval velocity model approaches zero. The key parameters can be seen from the residual moveout that oscillates around zero (zero residual moveouts) and gathers depth which shows a flat event. Also, targets can also be seen from the cross-section of PSDM produced. In this study, five iterations were performed.
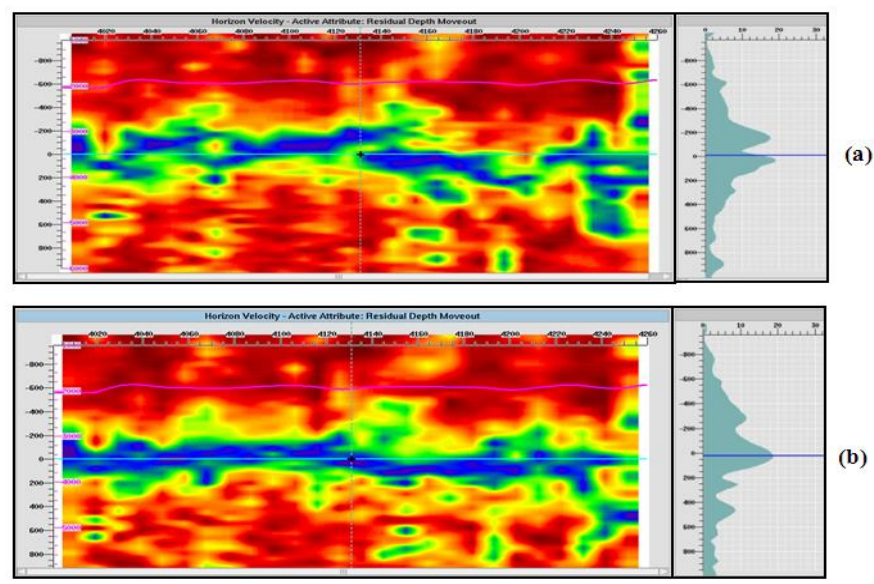

Figure 7 (a) Semblance residual moveout from the initial interval velocity model, (b) residual semblance moveout from the final interval velocity model after the 5th iteration, for horizon 2

Figure 7 is the residual moveout initial semblance image and after the 5th iteration in the second 
analysis of the residual moveout horizon. Figure 7 shows that the resulting slides are oscillating around zero after the 5th iteration, which indicates that the error in the interval velocity model is getting smaller. Figure 8, Figure 9, and Figure 10, respectively, show residual semblance moveout before and after tomography, the final interval velocity model, depth section, and collecting depth after the fifth tomography (last iteration) is performed.

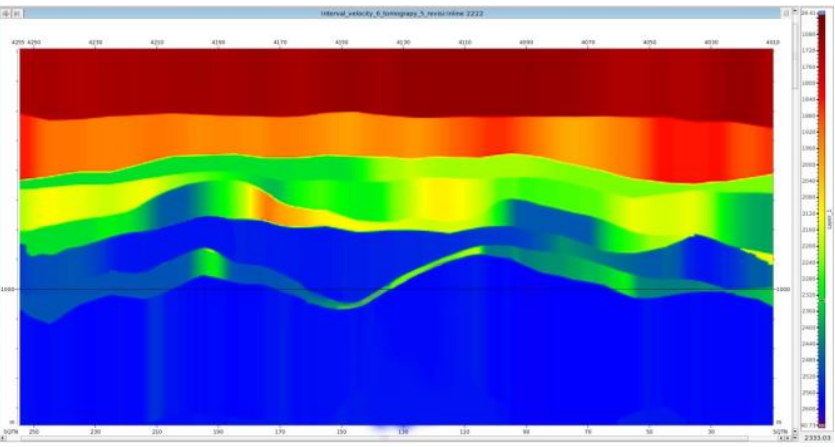

Figure 8 Model of latter interval velocity inline 2222, after fifth iteration tomography

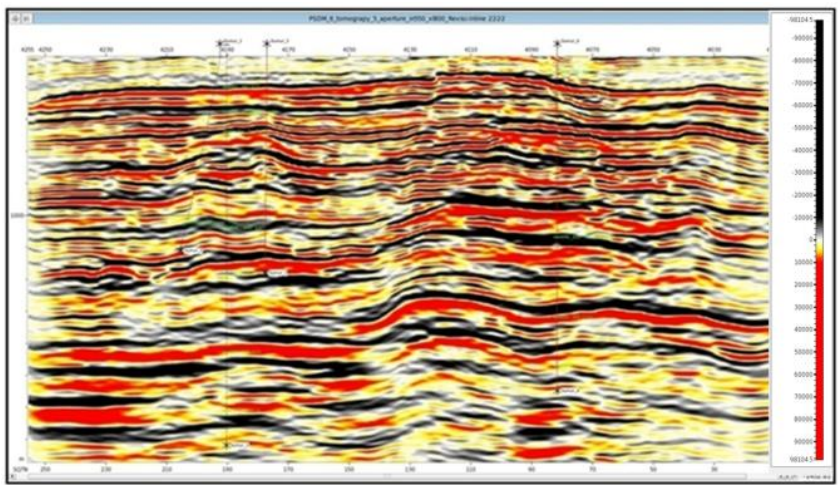

Figure 9 Depth section inline 2222 results of running PSDM using the final interval velocity model (5th iteration)

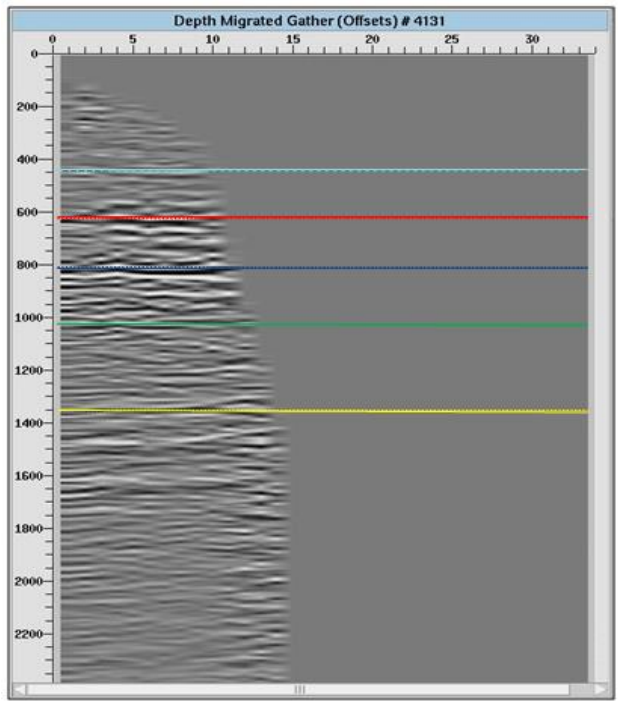

Figure 10 Final depth gathers after running the 5th tomographic PSDM on CRP 4131, and it appears that the events are flat
In the analysis of depth gathering, the results of PSDM are done by looking at events at the collecting depth whether it has shown a relatively flat event or not. The application of an inaccurate velocity model will result in a non-flat collection or residual moveout (difference in travel time between near and far offset). There are two types of residual moveout, namely the residual moveout is positive (the event is bent down) and the residual moveout is negative (upward bending event), as in Figure 11. Residual moveout positive indicates that the velocity used is too high (Figure 11a), while residuals moveout negatively indicates that the velocity used is too low (Figure 11b). The existence of a residual moveout means that events originating from the same reflection point do not have the same depth, should events originating from the same reflection point have the same depth at the depth gathers. This is based on the concept of PSDM which maps each event that comes from the same reflection point at the actual depth or position (if the velocity model used is accurate). Improvement of the interval velocity model is done until events are obtained at relatively flat (collecting) depth.

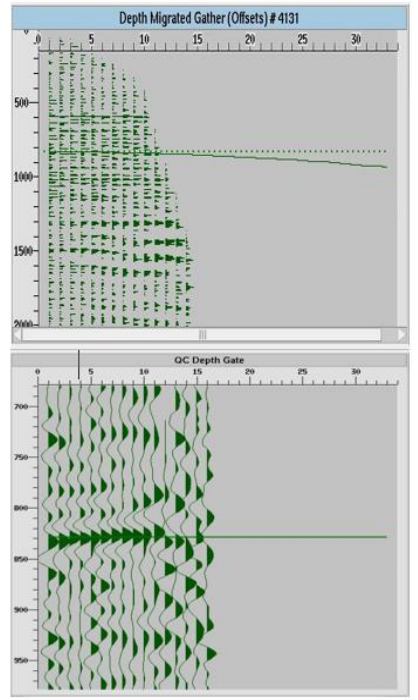

(a)

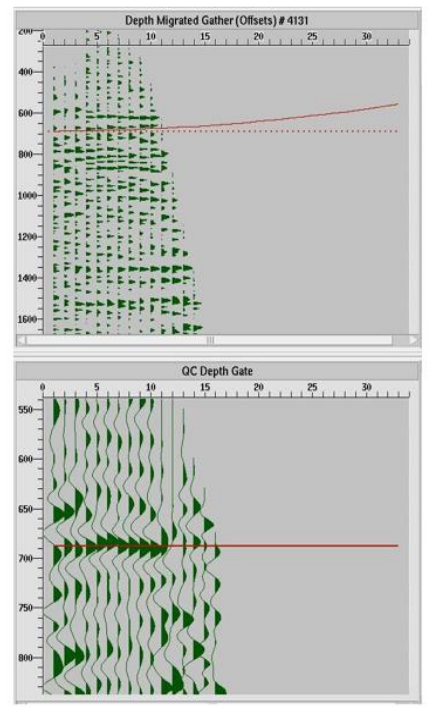

(b)
Figure 11 (a) The positive residual moveout (event bent downward), which indicates that the velocity used is too high, (b) a negative residual moveout (event bent upward), which indicates that the velocity used is too small

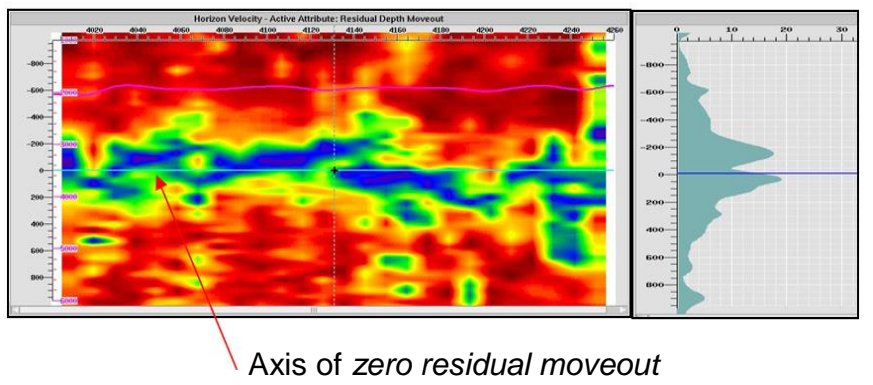

Figure 12 Semblance residual moveout which still shows an error in the initial interval velocity model 
The identification of interval velocity model errors with analysis of residual moveout semblance is calculated from depth gathers. Gathers that contain residual moveout will produce semblance, whose maximum value (Semblance peak) is not on the zero-residual moveout axis. This means, if Gathering has coincided with the zero residual moveouts (gathers flat) axis, then Gathering does not contain residual moveout. Figure 12 gives an example of a residual moveout semblance that is not on the residual moveout axis after the initial velocity interval model at horizon 2 is applied.

The residual moveout Semblance analysis is used for the PSDM depth image analysis stage along with the marker (well data). The incompatibility between depth image maps and reflectors that are related to the boundary layer in the depth image can be seen when both are displayed in a section (overlay), that is, the layer boundary at the interval velocity model will deviate from the reflector (layer boundary) it represents at the resulting depth image. Other errors can be identified from well markers that have not been tied to the horizon; this process is known as a well seismic tie. Well seismic tie is the work of putting a seismic horizon (on a time scale) at the actual depth position so that it can be correlated with other geological data and plotted on a depth scale by moving well data into seismic data so that its placement will be known. In geology, this binding aims to determine the top formation studied in the seismic cross-section.

Updating of the interval velocity model is done iteratively until the velocity interval, and depth image models are produced accordingly. The interval velocity model generated from the repair process with the horizon-based tomography method will be used again as a PSDM input; this process will continue to be repeated until a flat collecting is obtained.

\subsection{CONCLUSION}

In this study, the residual moveout analysis successfully applied to find errors at depth gathers due to the application of an inaccurate interval velocity model. Errors in this interval velocity model can be seen in the residual moveout semblance which is still far from zero residual moveouts. This residual moveout is then used to update the interval velocity model. Analysis of residual moveout and the process of updating the velocity model is done iteratively until the velocity interval and depth image models produced are consistent or appropriate.

\section{Acknowledgement}

We are grateful to Politeknik Negeri Batam, Universitas Gadjah Mada, Indonesia and Universiti Teknologi Malaysia for supporting this research

\section{References}

[1] Kret, K., Ikeda, T., \& Tsuji, T. 2019. Grid-search Inversion Based on Rock Physics Model for Estimation of Pore Geometry and Grain Elastic Moduli: Application to Hydrothermal Ore Deposits and Basalt. Exploration Geophysics. 50(1): 1-11. DOI: https://doi.org/10.1080/08123985.2018.1548605.

[2] Alkhalifah, T. 2003. Tau Migration and Velocity Analysis: Theory and Synthetic Examples. Geophysics. 68(4): 13311339. DOI: https://doi.org/10.1190/1.1598126.

[3] Yilmaz, Ö. 2001. Seismic Data Analysis: Processing, Inversion, and Interpretation of Seismic Data. Society of Exploration Geophysicists DOl: https://doi.org/10.1190/1.9781560801580.

[4] Agosta, F., Alessandroni, M., Antonellini, M., Tondi, E., \& Giorgioni, M. 2010. From Fractures to Flow: A Field-based Quantitative Analysis of An Outcropping Carbonate Reservoir. Tectonophysics. 490(3-4): 197-213. DOI: https://doi.org/10.1016/j.tecto.2010.05.005

[5] Han, J., Lv, Q., Gu, B., Yan, J., \& Zhang, H. 2019. 2D Anisotropic Multicomponent Gaussian-Beam Migration Under Complex Surface Conditions. Geophysics. 85(2): 188.

DOI: https://doi.org/10.1190/geo2018-0841.1.

[6] Irawan, S., \& Khoirunnisa, H. 2017. Identification of Reservoir Thickness and Estimation of Hydrocarbon Reservation used the Pre-Stack Depth Migration (PSDM) in Cikung Area. Geospatial Information. 1(1). DOI: https://doi.org/10.30871/jagi.v1i01.329.

[7] Irawan, S., Sismanto, S., \& Sukmatiawan, A. 2014. Applying the Horizon Based Tomography Method to Update Interval Velocity Model, Identify The Structure of Pre-Stack Depth Migration 3D and Estimate The Hydrocarbon Reserve In SBI Field of North West Java Basin. Jurnal Teknologi. 69(6). DOI: https://doi.org/10.11113/jt.v69.3240.

[8] Liu, J. Y., Xu, W., Chen, S. M., Guan, X. W., \& Pei, J. Y. 2020. Application of Viscoelastic Media Prestack Depth Migration in Unconventional Exploration Area. SEG 2019 Workshop: Mathematical Geophysics: Traditional vs Learning, Beijing, China, 5-7 November 2019. 135-138. Society of Exploration Geophysicists.

DOI: https://doi.org/10.1190/iwmg2019_34.1.

[9] Spadavecchia, E., Varvara, I., Cella, G., \& Magistroni, C. 2018. Challenges in Imaging a Highly Heterogeneous Carbonate Reservoir in a Complex Geological Setting. SPE Annual Caspian Technical Conference and Exhibition. Society of Petroleum Engineers. DOI: https://doi.org/10.2118/192574-RU.

[10] Li, J., Huang, J., Li, Y., \& Junjun WU, Y. C. 2018. Walkaway VSP Reflection Tomography Modeling and Prestack Depth Migration Imaging. In International Geophysical Conference, Beijing, China, 24-27 April 2018. 1339-1342. Society of Exploration Geophysicists and Chinese Petroleum Society. DOI: https://doi.org/10.1190/IGC2018-328.

[11] Widiyantoro, S., Gorbatov, A., Kennett, B. L. N., \& Fukao, Y. 2000. Improving Global Shear Wave Traveltime Tomography Usingthree-Dimensional Ray Tracing and Iterative Inversion. Geophysical Journal International. 141 (3): 747-758.

DOI: https://doi.org/10.1046/j.1365-246x.2000.00112.x.

[12] Woodward, M. J., Nichols, D., Zdraveva, O., Whitfield, P., \& Johns, T. 2008. A Decade of Tomography. Geophysics. 73(5): VE5-VE1 1. DOI: https://doi.org/10.1190/1.2969907

[13] Xue, Z., Sun, J., Fomel, S., \& Zhu, T. 2018. Accelerating Fullwaveform Inversion with Attenuation Compensation. Geophysics. 83(1): A13-A20. DOI: https://doi.org/10.1190/geo2017-0469.1. 
[14] Abbasi, M., \& Gholami, A. 2018. Automatic Nonhyperbolic Velocity Analysis by Polynomial Chaos Expansion. Geophysics. 83(6): U79-U88. DOI: https://doi.org/10.1190/geo2017-0478.1.

[15] Zhang, H. 2018. Analysis of Factors Affecting Residual Moveout Picking and Solutions. International Journal of Oil, Gas and Coal Engineering. 6(6): 150. DOl: https://doi.org/10.11648/j.ogce.20180605.15.

[16] Di Maio, R., De Paola, C., Forte, G., Piegari, E., Pirone, M. Santo, A., \& Urciuoli, G. 2020. An Integrated Geological, Geotechnical and Geophysical Approach to Identify Predisposing Factors for Flowslide Occurrence. Engineering Geology. 105473. DOI: https://doi.org/10.1016/j.enggeo.2019.105473.

[17] Delgado, M. A., \& Robinson, P. M. 1992. Nonparametric and Semiparametric Methods for Economic Research Journal of Economic Surveys. 6(3): 201-249. DOI: https://doi.org/10.1111/j.1467-6419.1992.tb00151.x.

[18] Zhao, Y., Wang, Y., Wang, W., Tang, L., Liu, Q., \& Cheng, G. 2019. Modeling of Rheological Fracture Behavior of Rock Cracks Subjected to Hydraulic Pressure and Far Field Stresses. Theoretical and Applied Fracture Mechanics. 101: 59-66.
DOI: https://doi.org/10.1016/j.tafmec.2019.01.026.

[19] Takougang, E. M. T., Bouzidi, Y., \& Ali, M. Y. 2019. Characterization of Small Faults and Fractures in a Carbonate Reservoir Using Waveform Inversion, Reverse Time Migration, and Seismic Attributes. Journal of Applied Geophysics. 161: 116-123.

DOI: https://doi.org/10.1016/j.jappgeo.2018.12.012.

[20] Leite, L. W. B., \& Vieira, W. W. S. 2019. Automatic Seismic Velocity Analysis Based on Nonlinear Optimization of the Semblance Function. Journal of Applied Geophysics. 161: 182-192. DOI: https://doi.org/10.1016/j.jappgeo.2018.12.015

[21] Wu, Z. W., Wu, Y. J., Guo, S., \& XU, M. H. 2018. Q-factor Estimation in CMP Gather and the Continuous Spectral Ratio Slope Method. Applied Geophysics. 15(3-4): 481-490. DOI: https://doi.org/10.1007/s11770-018-0672-y.

[22] Schneider, J. F. 2019. Horizon Oriented Residual Prestack Migration to Zero Offset. SEG Technical Program Expanded Abstracts 2019. 4312-4316. Society of Exploration Geophysicists. DOI: https://doi.org/10.1190/segam2019-3216095.1. 